\title{
Erratum to: High fracture probability predicts fractures in a 4-year follow-up in women from the RAC-OST-POL study
}

\author{
W. Pluskiewicz ${ }^{1}$ P. Adamczyk ${ }^{2}$ - A. Czekajło ${ }^{3} \cdot$ W. Grzeszczak ${ }^{4}$ B. Drozdzowska ${ }^{5}$
}

Published online: 23 July 2015

(C) International Osteoporosis Foundation and National Osteoporosis Foundation 2015

\section{Erratum to: Osteoporos Int}

DOI 10.1007/s00198-015-3196-9

The last sentence of the abstract, repeated as the last sentence of the full text, was misleadingly worded. The correct sentence is as follows:

\begin{abstract}
A number of clinical risk factors and a prior fracture, rheumatoid arthritis, femoral neck T-score, and falls were independently associated with an increased incidence of fractures.
\end{abstract}

The authors apologize for this error and for any inconvenience caused.

The online version of the original article can be found at http://dx.doi.org/ 10.1007/s00198-015-3196-9.

W. Pluskiewicz

osteolesna@poczta.onet.pl

1 School of Medicine with the Division of Dentistry, Metabolic Bone Diseases Unit, Department and Clinic of Internal Diseases, Diabetology and Nephrology, Medical University of Silesia in Katowice, Maja 13/15 street, 41-800 Zabrze, Poland

2 School of Medicine with the Division of Dentistry, Department and Clinic of Pediatrics, Medical University of Silesia in Katowice, Zabrze, Poland

3 Department of Nephrology, Wodzisław, Poland

4 School of Medicine with the Division of Dentistry, Department and Clinic of Internal Diseases, Diabetology and Nephrology, Medical University of Silesia in Katowice, Zabrze, Poland

5 School of Medicine with the Division of Dentistry, Department of Pathomorphology, Medical University of Silesia in Katowice, Zabrze, Poland 